\section{Endoscopic gastroesophageal mucosal flap valvuloplasty with anti-reflux potential}

The lower esophageal sphincter (LES), the crural diaphragm, and the gastroesophageal flap valve (GEFV) are key components of the esophagogastric junction (EGJ) and the anti-reflux barrier. Currently available endoscopic techniques to treat gastroesophageal reflux disease (GERD) include suturing devices, transmural staplers, prosthetic implantation, and radiofrequency ablation; all aimed at reinforcing the LES [1]. Although reflux can occur when increases in intra-abdominal pressure surpass the hypotensive LES pressure, most reflux takes place during spontaneous relaxations of the EGJ, which are known as "the transient LES relaxations" (TLESRs) [2].

The GEFV and the associated acute angle of His can serve as a mechanical barrier by compressing the proximal stomach against the intra-abdominal esophagus, thereby limiting EGJ opening. While disruption of the GEFV structures in patients with GERD, particularly those with a hiatal hernia, has been assessed and graded for many years [3], recent functional anat- omy analysis using magnetic resonance imaging (MRI) and high resolution manometry (HRM) has demonstrated that, compared with healthy subjects, patients with GERD have a wider insertion angle, which might compromise the function of the GEFV [4]. Therefore, the GEFV and its associated angle of His could be both an anatomical culprit and a therapeutic target for GERD. Here, we describe our recently developed endoscopic mucosal flap valvuloplasty technique to build/elongate the GEFV.

The study, using both preoperative anesthesia and postoperative euthanasia, was conducted in a porcine model. The endoscopic procedure was performed using a single-channel gastroscope (RS110; Pentax, Tokyo, Japan) with a transparent cap (MH-588; Olympus, Tokyo, Japan) attached.

We began the procedure by making a 2-cm longitudinal mucosal incision using a Dual knife (KD-650l; Olympus) after submucosal injection of saline and methylene to form an entry site about $4 \mathrm{~cm}$ above the EGJ ( $\bullet$ Fig. $1 \mathbf{a}$ and $\bullet$ Fig. 2 a). A submucosal tunnel was then dissected from the esophagus, through the cardia, extending within the gastric submucosal space, eventually to a site $5 \mathrm{~cm}$ distal to the EGJ ( $\bullet$ Fig. $\mathbf{1} \mathbf{b}$ and $\bullet$ Fig. 2 b) [5]. The gastric submucosal tunnel was bilaterally extended using endoscopic submucosal dissection (ESD) to create a $135^{\circ}$ fanshaped submucosal space centered on the cardia with a radius of $5 \mathrm{~cm}$. This space separated the covering mucosa and part of the submucosal layer from the underlying muscularis propria ( $\mathbf{F i g . 1 b}$ and - Fig.2c). An electric heating forceps was used both for coagulation of visual bleeding and for prophylactic hemostasis, as the fundal submucosal space is richly vascularized.

The endoscope was subsequently withdrawn from the tunnel, advanced into the gastric lumen, and retroflexed to detect the elevated, fan-shaped, detached fundal mucosa, which was stained in blue ( $\bullet$ Fig.2c). After the submucosa had been injected and cautery markings made, the detached fundal mucosa, with the exception of its proximal part near cardia, was circumferentially incised along its verge using an IT-knife (KD611L; Olympus) ( $\bullet$ Fig. $1 \mathrm{c}$ and $\bullet$ Fig. 2 d). Because of the self-elasticity of the muscularis mucosa, the free-hanging fundal
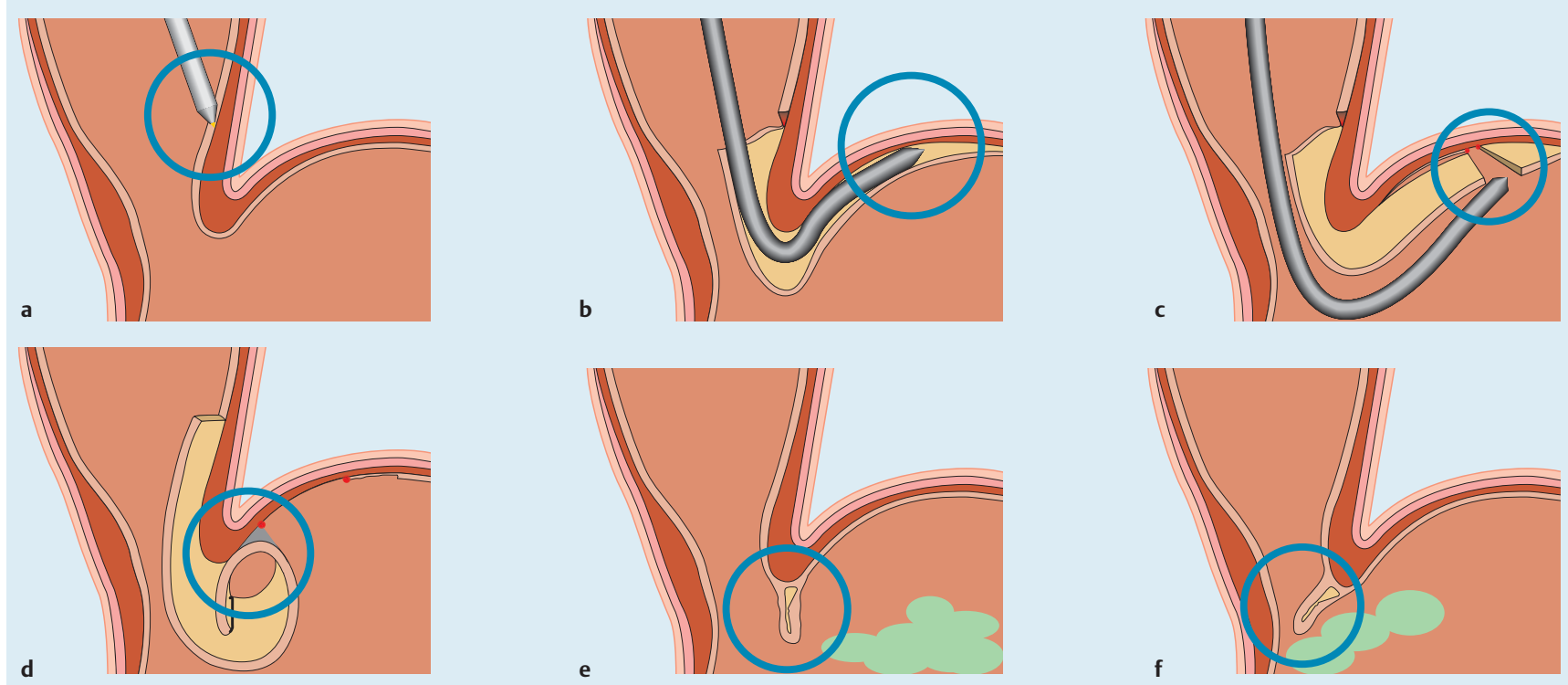

Fig. 1 Schematic illustrating the procedure of endoscopic gastroesophageal mucosal flap valvuloplasty. a A 2-cm longitudinal mucosal incision is made approximately $4 \mathrm{~cm}$ above the gastroesophageal junction (GEJ) as a mucosal entry into the submucosal space. $\mathbf{b}$ A transcardiac submucosal tunnel is created to a site $5 \mathrm{~cm}$ distal to the GEJ. c A retroflexed endoscope is used to view the elevated, blue-stained fan-shaped gastric fundal mucosa, which is incised along its verge, with the exception of its proximal edge, then detached from the gastric luminal wall. $\mathbf{d}$ The incised mucosa curls spontaneously to form a doublelayered mucosal flap. $\mathbf{e}$ The initial esophageal mucosal entry is closed with hemostatic clips, leaving a double-layered mucosal flap present at the GEJ. $\mathbf{f}$ The flap valve is thin and pliable so that it can prevent regurgitation of gastric contents into the esophagus. 


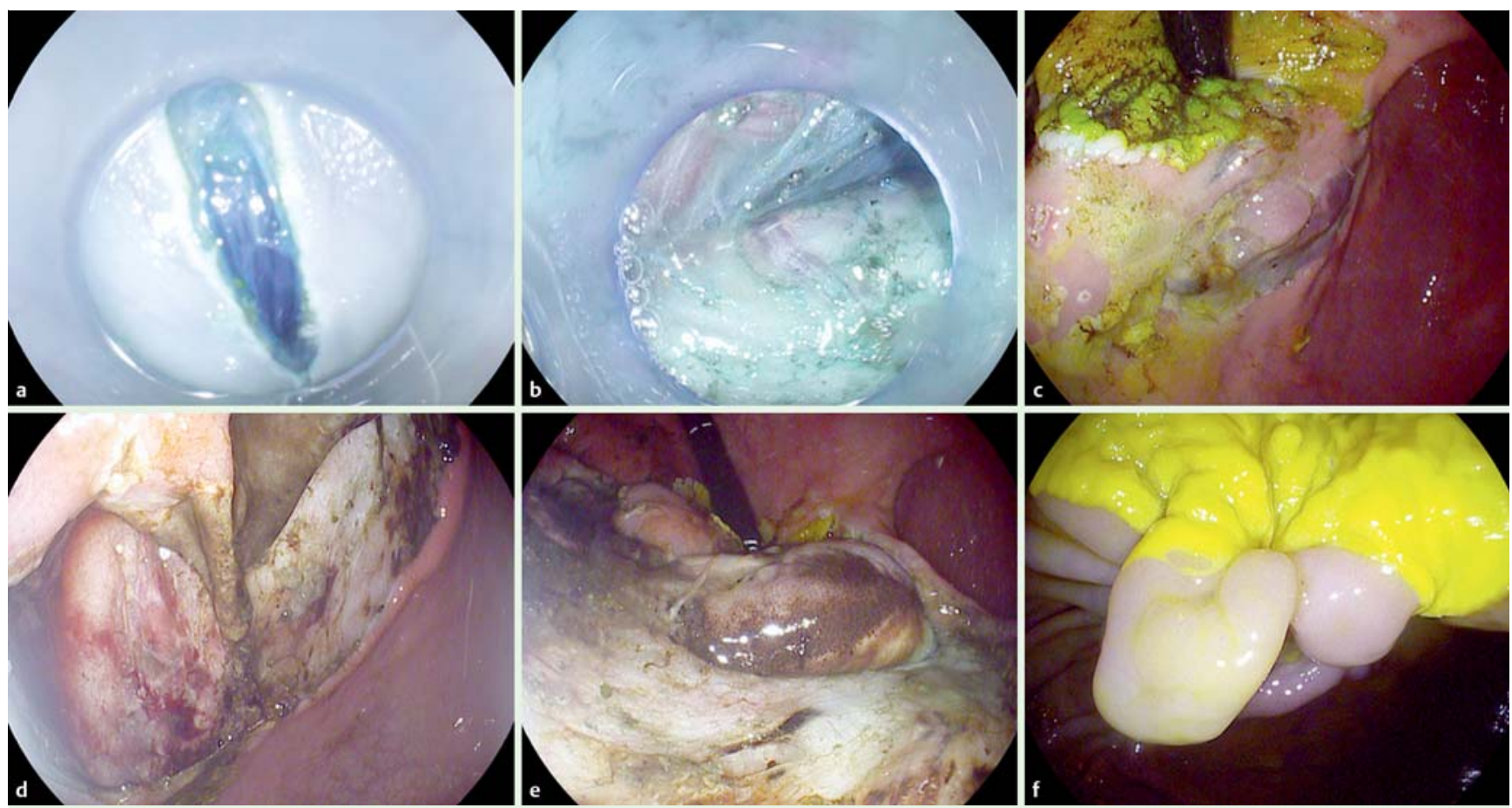

Fig. 2 Endoscopic pictures of the gastroesophageal mucosal flap valvuloplasty procedure showing: a the initial mucosal incision in the lower esophagus; b creation of a transcardiac submucosal tunnel; $\mathbf{c}$ in retroflexed view, the elevated fan-shaped gastric fundal mucosa stained blue; $\mathbf{d}$ incision of the fan-shaped mucosa along its verge, excluding its proximal edge; $\mathbf{e}$ the detached mucosa curling spontaneously toward the cardia $\mathbf{f}$ the final gastroesophageal mucosal flap.

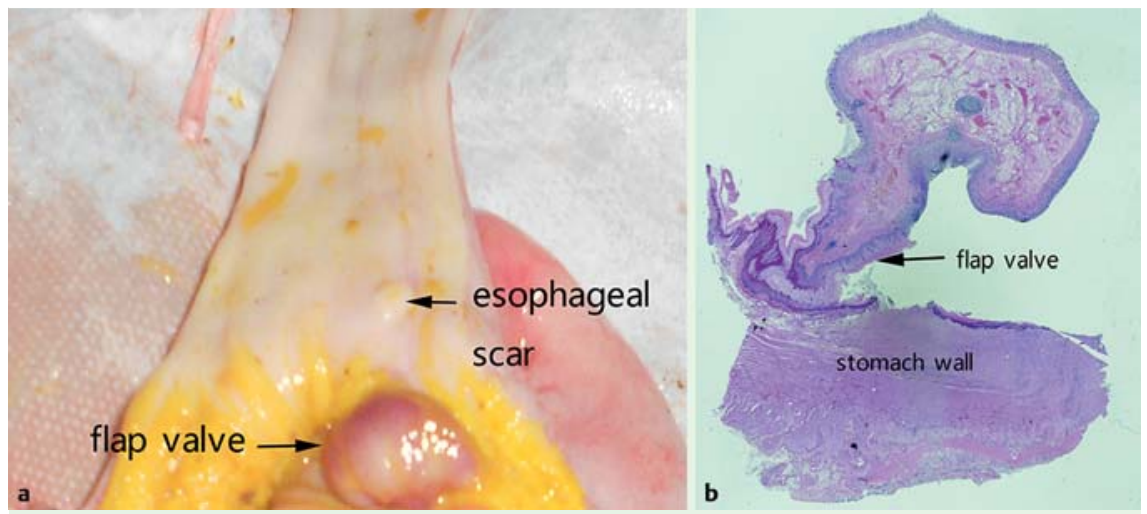

Fig. 3 The created mucosal flap at necropsy. a Macroscopic appearance after gastrostomy. b Histological appearance showing the sandwich-like structure of the flap valve with covering mucosal layers and in between these a submucosal layer with blood vessels.

mucosa automatically curled toward the cardia to form a gastroesophageal mucosal flap ( $\bullet$ Fig. $\mathbf{1 d}$ and $\bullet$ Fig. 2 e). Therefore, the mucosal flap was composed of a double layer of mucosa with submucosal tissue sandwiched in between. The initial esophageal mucosal entry was eventually closed with hemo clips (DLC-D-135; Microtech, Aurora, Colorado, USA) ( Fig. 1 e, - Fig. $1 \mathrm{f}$ and $\odot$ Fig. 2f).

During 3 weeks of follow-up after the procedure, the pig appeared to do well, with no evidence of fever or dysphagia. Endoscopic re-examination showed that both the incision in the esophagus and the mu- cosal ulcer on the gastric fundus had healed, and a living, prominent mucosal flap was observed at the EGJ.

At necropsy, the size of the flap was $1.9 \mathrm{~cm} \times 1.5 \mathrm{~cm} \times 0.5 \mathrm{~cm}(\bullet \mathrm{Fig} .3 \mathrm{a}$ ). Pathological examination demonstrated the sandwich-like flap structure with the mucosal layers on either side and a submucosal layer in the middle; small blood vessels could be observed ( $\bullet$ Fig. 3 b). Therefore, this endoscopic valvuloplasty procedure appears safe and feasible.

This procedure was developed on the basis of advanced endoscopic resection techniques and has the potential to re- store the GEFV function in patients with GERD. While current tests, such as measurement of the LES/gastric yield pressure or esophageal $\mathrm{pH}$, are not suitable for evaluating GEFV function in a porcine model, ex vivo gastric perfusion tests or $\mathrm{pH}$ monitoring in primate animals could be applied in the future.

Endoscopy_UCTN_Code_TTT_1AO_2AJ

Competing interests: None

\section{Jiaoyang Lu' ${ }^{1,2, *}$, Yanmei Li ${ }^{1, *}$, Taotao Jiao ${ }^{3}$, Ying Liu ${ }^{1}$, Yanan Wang', Yatian Wang', Minhua Zheng ${ }^{2}$, Xuefeng Lu ${ }^{1}$}

${ }^{1}$ Department of Gastroenterology, Qilu Hospital, Shandong University School of Medicine, Shandong, China 2 Department of General Surgery, Ruijin Hospital, Shanghai Jiao Tong University School of Medicine, Shanghai, China ${ }^{3}$ Department of Statistics, Shandong Provincial Hospital, Shandong, China

\footnotetext{
* Jiaoyang Lu and Yanmei Li contributed equally to this work.
} 


\section{References}

1 Iqbal A, Salinas V, Filipi CJ. Endoscopic therapies of gastroesophageal reflux disease. World J Gastroenterol 2006; 12: 2641 - 2655

2 Bredenoord AJ, Pandolfino JE, Smout AJ. Gastro-oesophageal reflux disease. Lancet 2013; 381: 1933-1942

3 Hill LD, Kozarek RA, Kraemer SJ et al. The gastroesophageal flap valve: in vitro and in vivo observations. Gastrointest Endosc 1996; 44: $541-547$

4 Curcic J, Roy S, Schwizer A et al. Abnormal structure and function of the esophagogastric junction and proximal stomach in gastroesophageal reflux disease. Am J Gastroenterol 2014; 109: 658-667
5 Lu J, Zheng M, Jiao T et al. Transcardiac tunneling technique for endoscopic submucosal dissection of gastric fundus tumors arising from the muscularis propria. Endoscopy 2014; 46: $888-892$

\section{Bibliography}

DOI http://dx.doi.org/

10.1055/s-0042-111390

Endoscopy 2016; 48: E268-E270

(c) Georg Thieme Verlag KG

Stuttgart · New York

ISSN 0013-726X
Corresponding author

\section{Xuefeng Lu, MD}

Department of Gastroenterology

Qilu Hospital

Shandong University School of Medicine

Jinan

Shandong 250012

China

Fax: 86-531-82166095

lu0801shanyi@163.com 